\title{
'||-||||||||||||||||||||||||||||||||||||||||||||||||||||||||||||||||||||.
}

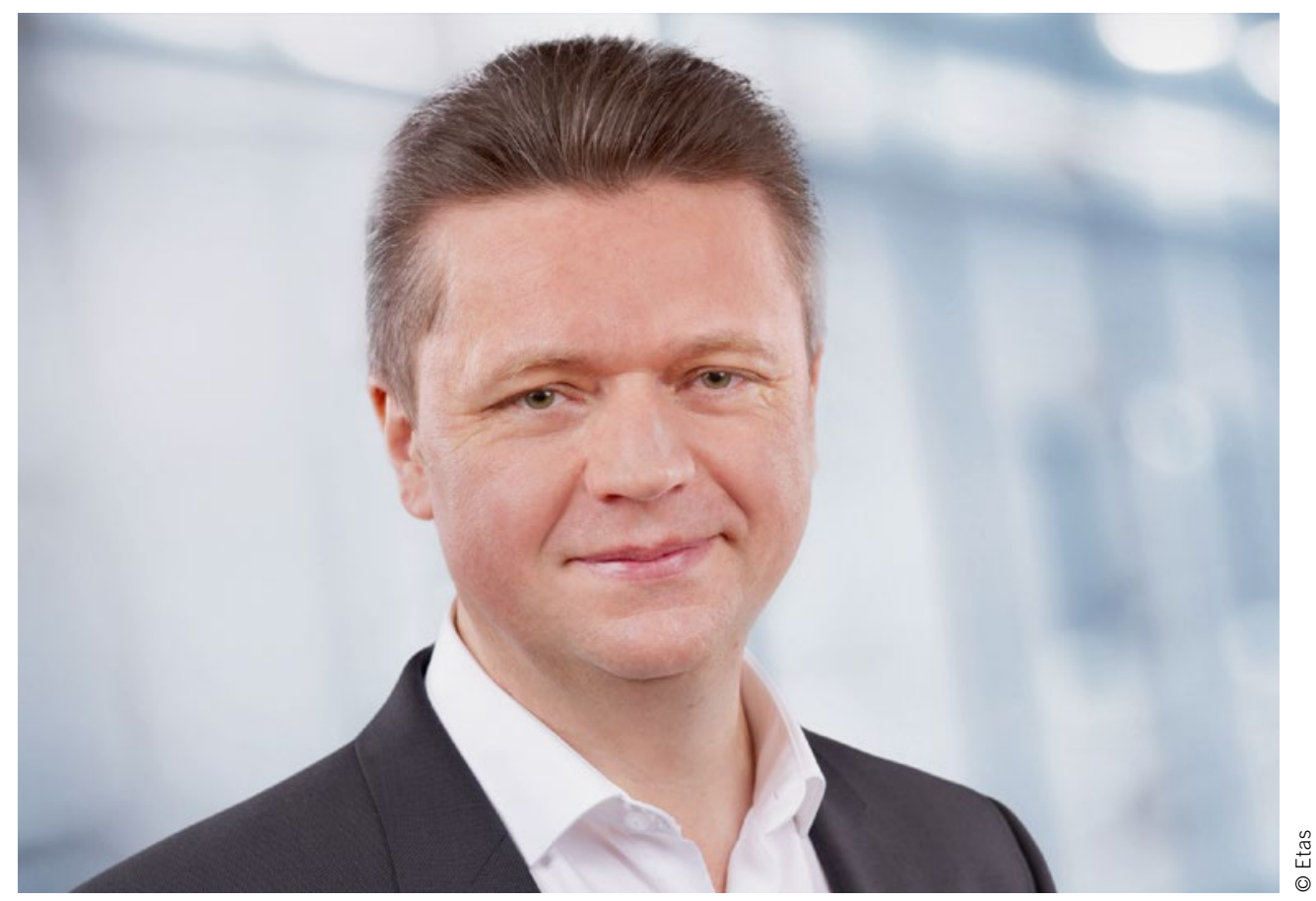

Günter Gromeier

Vice President der

Produktgruppe Automotive

bei Etas

\section{Abgestimmte Entwicklungslösungen sind der Schlüssel zum Erfolg}

Veränderung, Transformation und Ungewissheit sind die Begriffe, welche die aktuelle Situation in der Automobilindustrie wohl am besten beschreiben. E-Mobilität, Vernetzung, (teil-)automatisiertes Fahren, Mobilitätsservices, Automotive Apps, ... Die Liste der technischen Herausforderungen lässt sich beliebig fortsetzen. Was wird wie schnell auf den Markt kommen? Eines ist allen gemeinsam: Software spielt bei den meisten Innovationen die zentrale Rolle.

Der Veränderungsdruck ist enorm. Große Player aus der IT-Welt drängen auf den Markt. Neue Mobilitätsgeschäftsmodelle für Fahrzeuge im Feld gewinnen an Bedeutung. Viele Softwareprojekte werden von der Hardware abgekoppelt entwickelt. Zusätzlich gelten für Automotive Software höchste Sicherheitsanforderungen - besonders im ADAS/AD-Bereich. Es gilt also, innovative Software schnell, sicher und ökonomisch auf den Markt zu bringen und diese laufend im Feld zu aktualisieren. Agile Entwicklungsmethoden, virtuelle Validierung sowie Continuous Integration und Deployment (CI/CD) sind Voraussetzungen für den Erfolg. Betrachtet man die Entwicklung genauer, kann man unter anderem vier wichtige Bereiche identifizieren: funktionale Anwendungssoftware, Middleware für mikrocontroller- und mikroprozessorbasierte Hardware, holistische Lösungen für Cybersecurity und Entwicklungswerkzeuge für eine effiziente und sichere Entwicklung von komplexen Systemen mit großen Datenmengen, die schnellste Entwicklungszyklen ermöglichen; sei es am klassischen Entwicklerarbeitsplatz oder in der Cloud. Während die Anwendungssoftware für den OEM differenzierend ist, bieten Middleware, Security und Werkzeuge ein großes Potenzial für Standardisierung und gemeinsame Entwicklungsplattformen. Dies bietet neben Synergieeffekten auch ein höheres Maß an Sicherheit. Denn der Aufwand für den Nachweis der funktionalen Sicherheit und die Maßnahmen für durchgängige Security steigen überproportional zur Komplexität, dem Vernetzungsgrad und der Updatehäufigkeit der Software. Diese Aufgabe lässt sich nur gemeinsam bewältigen.

So können sich die OEMs und ihre Zulieferer auf die eigentlich differenzierende Anwendungsfunktionalität konzentrieren und schnell zum Ziel gelangen. Zentraler Aspekt für den Erfolg ist, dass Middleware, Security-Lösungen und Entwicklungswerkzeuge aufeinander abgestimmt sind. Nur so können die Entwicklungspartner eine hohe Effizienz erzielen. Geschwindigkeit und Umfang der Veränderungen in der Automotive-Softwareentwicklung waren noch nie so groß wie heute. Eine gute Zusammenarbeit sowie Partnerschaften sind dabei wichtiger denn je. Die Zeit der reinen Insellösungen ist definitiv vorbei. 